\title{
Factores endógenos y su relación con la permanencia irregular: una lectura desde los estudiantes
}

Recibido: 05/10/2015

Aceptado: 15/03/2016

\section{Endogenous factors and their relation with irregular permanence: A reading from students' perspective}

\author{
Carlos Gómez Cano, carlosgomez325@gmail.com \\ Verenice Sánchez Castillo,ve.sanchez@udla.edu.co \\ Ginna Tovar Cardozo,g.tovar@udla.edu.co \\ Universidad de la Amazonia, Colombia
}

\section{Resumen}

El fenómeno de permanencia irregular, entendido como el exceso de permanencia ininterrumpida de los estudiantes respecto a los tiempos establecidos en los planes de estudio, es un asunto que cuestiona la eficiencia y la calidad de los sistemas educativos. En el caso de la Facultad de Ciencias Contables, Económicas y Administrativas -FCCEA, de la Universidad de la Amazonia, este no ha sido objeto de procesos investigativos que permitan tomar o generar oportunidades de mejora. El presente estudio tuvo como objetivo analizar los factores endógenos que inciden en la permanencia irregular desde el sentir del estudiante. Para ello, se realizó un abordaje cuantitativo empleando la encuesta como instrumento para la recolección de la información. Dentro de los hallazgos identificados, se tiene que los factores con mayor incidencia en la permanencia irregular son el rendimiento académico y la docencia, razón por la cual se sugirió la adopción de políticas institucionales que propendan por la erradicación de esta situación.

Palabras claves: Permanencia, graduación, institución, educación, endógeno.

\section{Abstract}

Irregular permanence is best defined as students' interrumpted stay from pre-stablished timing to persevere in their carreer's study plan. However, the influence of factors leading to irregular permanence in students and their relation to quality of education and eficiency has not yet been undertaken as a matter of research by Social studies, accountancy, management and economical faculty of Amazonia University (FCCEA). The purpose of this study was to analize endogenous factors and their relation with irregular permanence from student's point of view. Thus, a quantitative methologogial research approach that focused on questionaries used to gather data was employed throughout the study. The findings revealed that among the factors identified to be leading to students' irregular permanence, academic performance and teaching practices resulted to be the highest influencial factors on students' irregular permanence, suggesting the need adopting new institutional policies that would erradicate this phenomenom.

Keywords: Permanence, promotion, insitution, education, endogenous factors. 


\section{Introducción}

\section{Breve contexto de la educación superior en Colombia}

Para la UNESCO (2014), la educación es un medio indispensable para el desarrollo integral de las personas, reconociéndola también como un elemento clave para la construcción de una sociedad igualitaria y desarrollada (Sánchez, Gómez y Polonía, 2016). Esta premisa no es ajena a las realidades de Colombia, pues desde la Constitución Política de 1991, se estableció que:

La educación es un derecho de la persona y un servicio público que tiene una función social; con ella se busca el acceso al conocimiento, a la ciencia, a la técnica, y a los demás bienes y valores de la cultura (Art. 67).

Este escenario brinda a la educación, además de una gran importancia por estar considerada a nivel constitucional, un rol protagónico en la construcción de una sociedad integral (Gómez, Sánchez y Rivera, 2016).

Respecto a la educación superior, los esfuerzos del congreso de Colombia por regular e implementar el mandato constitucional se materializaron a través de la Ley 30 de 1992, la cual tiene como propósito organizar el servicio público de la educación superior en Colombia. La filosofía de esta actuación legislativa es el fomento a la educación superior, garantía de la calidad en la prestación del servicio, autonomía administrativa para las instituciones educativas y disposiciones de control para garantizar la integralidad del proceso.

Bajo este entendido y siguiendo a Misas (2004), se hace evidente la responsabilidad que se ha encomendado a la educación superior para la formación de ciudadanos como agentes de desarrollo y de solución de problemas del entorno, amén de mejorar las condiciones de vida de la sociedad. Adicionalmente, el autor expresa que:

La importancia estratégica de la educación superior se hace visible tan pronto como se reconocen los efectos de la incorporación de la ciencia y la tecnología, y de la reflexión elaborada sobre los fines, en los procesos de trabajo, en la producción de la riqueza material y simbólica y en el desarrollo de la organización social (p. 14).

Estas realidades no han sido ajenas para los líderes gubernamentales, pues la educación siempre se ha considerado como un asunto de vital importancia. Tal es el caso del actual Plan Nacional de Desarrollo 2014-2018, el cual considera la educación como eje estratégico en la agenda de la gestión pública nacional. Se concibe a la educación «como el más poderoso instrumento de igualdad social, pues no solo nivela las oportunidades de la personas, sino que abre puertas de progreso» (DNP, 2015, p. 25). Este documento plantea ambiciosas metas respecto a la cobertura, fomento, calidad, financiamiento, articulación y desarrollos en torno a las políticas de educación superior, esfuerzos que apuntan a la transformación integral de la cultura social, escalón indispensable en el propósito de construir una paz estable y duradera.

a Cobertura y permanencia académica: grandes retos de las universidades: En el apartado anterior se abordó la importancia de la educación superior como una poderosa herramienta de intervención de los contextos sociales del país, sin embargo, esta sublime realidad parece desvanecerse cuando se miran las crudas estadísticas de cobertura y permanencia en el sistema educativo. Estas denotan claramente la ausencia de una capacidad institucional que permita vincular a todos los jóvenes 
que egresan de la formación media. Sobre el particular, la Universidad Abierta para Adultos - UAPA (2009) comenta:

Las estadísticas educativas de Colombia al respecto son preocupantes. De acuerdo con datos del año 2004, de cada 100 estudiantes que ingresaron a la escuela, sólo 47 lograron terminar bachillerato. De éstos, sólo 27 ingresaron a la educación superior. Y, del escaso número de quienes ingresaron, sólo un poco más de la mitad terminaron exitosamente sus estudios y se titularon en cualquiera de las modalidades de educación superior (técnica, tecnológica o universitaria) (p. 18).

Por su parte, Melo, Ramos, y Hernández, (2014), sostienen que Colombia enfrenta grandes retos en términos de ampliación de cobertura, pues pese a los grandes esfuerzos realizados en las últimas décadas, los indicadores siguen estando por debajo de la media internacional, lo que deja en evidencia un largo camino por recorrer en materia de ingreso y permanencia en los sistemas de educación superior. Además, los autores expresan que:

Durante los últimos 20 años, el acceso a la educación superior aumentó a un mayor ritmo, lo que se tradujo en una tasa de cobertura bruta de $24,0 \%$ en el año 2000 y de $42,4 \%$ al final de 2012 . Durante este periodo, la población matriculada a nivel de pregrado ascendió de 487.448 estudiantes en 1990 a 1.841.282 en 2012, lo que significó una ampliación de los cupos cercana a 278\% (p. 10).

No obstante, desde una perspectiva internacional, la tasa de cobertura de Colombia es relativamente baja cuando se compara con países desarrollados como Estados Unidos, Finlandia, España, Nueva Zelandia, Australia, Noruega y con un grupo de países latinoamericanos como Argentina, Chile, Cuba, Uruguay y Puerto Rico, cuyas tasas de cobertura superan el 60\% (p. 12).

Estos escenarios han generado una serie de esfuerzos de autoridades como el Ministerio de Educación Nacional, universidades y organizaciones en torno al tema educativo, pues dadas las limitaciones existentes para el acceso a la educación superior, además de buscar caminos que permitan ampliar estos indicadores de cobertura, es necesario que exista una adecuada permanencia en las universidades, es decir, que la promoción académica - sin de dejar de lado la calidad - corresponda a lo proyectado, evitando que lo estudiantes excedan los tiempos definidos dentro de su plan de estudios al interior de la universidad.

Sobre el particular, el Ministerio de Educación Nacional - MEN (2009) expone que a partir de resultados de procesos de investigación realizados en el país, se determinó de que «las estrategias de retención deberían centrarse en aquellas variables que aparecen como fuertes determinantes en la disminución del riesgo de deserción y del aumento en los niveles de graduación y que además pudieran ser intervenidas directamente por la institución» (p. 34). Es decir, que las universidades deben generar políticas académicas que fomenten una adecuada estancia de los estudiantes, buscando mitigar cualquier fenómeno que interfiera con su cometido.

\section{Aproximaciones, el fenómeno de la permanencia irregular}

Al observar los panoramas descritos en los acápites anteriores, se hace evidente que las universidades deben generar esfuerzos de intervención que, sin atentar contra la calidad, permitan un efectivo trasegar de los estudiantes por el sistema educativo. Para el MEN (2013), una de las estrategias prioritarias para la educación superior en Colombia gira en torno a «continuar con la labor de fortalecimiento de la capacidad de las instituciones de educación superior para diseñar, ejecutar y evaluar acciones 
dirigidas al fomento de la permanencia y graduación estudiantiles» (p. 6).

Bajo este entendido, las universidades deben someterse a constantes procesos de autoevaluación, los cuales permitirán generar una radiografía institucional, detectando de esta manera cualquier anomalía en el sistema que atente contra sus propósitos misionales. La permanencia irregular, fenómeno tipificado en aquellos estudiantes que, sin caer en la problemática de la deserción, están vinculados a la universidad por más del tiempo estipulado dentro de sus respectivos planes de estudio, se ha convertido en un tema controvertido en los últimos tiempos, pues cuestiona a las instituciones sobre sus estrategias de seguimiento y promoción. En palabras de Gómez, Sánchez y Jiménez (2016):

La permanencia irregular se convierte en un fenómeno de incidencia negativa sobre la vida académica de los estudiantes y la dirección de las IES, pues son varios sus impactos e implicaciones al interior del sistema educativo. Uno de ellos, por ejemplo, está relacionado con la oferta académica universitaria, pues un estudiante que permanezca por un tiempo mayor al establecido puede llegar a reducir la posibilidad de ingreso de otro al sistema (p. 291).

De esta manera, el presente escrito expone los resultados de un proceso de investigación realizado en la Universidad de la Amazonia, específicamente en la Facultad de Ciencias Contables, Económicas y Administrativas. En el se detalla, a juicio de los estudiantes, la incidencia de factores endógenos de la institución en su condición de permanencia irregular. Para tal efecto, en la primera fase de esta investigación, luego de un robusto ejercicio de revisión documental, se establecieron seis factores con alto riesgo de incidir en la permanencia irregular, a saber: administración, currículo, rendimiento académico, medios educativos, bienestar universitario y docentes.

\section{Marco teórico}

Las causas de la permanencia irregular son atribuidas a múltiples factores, algunos de procedencia exógena, es decir, que no están relacionados directamente con la Universidad, como las condiciones socioeconómicas y los contextos geográficos e históricos (Dupéré, Leventhal, Dion, Crosnoe, Archambault, y Janosz, 2014). Condiciones económicas y culturales (Cuchacovich, 2011); incluyendo factores exógeno-materiales y exógeno-culturales (Carrión, Sanmartín, Fernández, 2014). Sin embargo, el objetivo de la presente investigación es analizar los factores endógenos que causaron permanencia irregular en los estudiantes de pregrado presencial de la FCCEA, durante el periodo 20102014. Es decir, aquellos factores que están ligados a la Institución. situación que según Ambroggio (2012), esta situación demanda la total atención de los involucrados en el proceso de la educación superior, toda vez que la no atención de las irregularidades en la permanencia estudiantil tiene un fuerte impacto sobre las distintas áreas de funcionamiento de cada unidad académica. Conforme a lo anterior, conviene retomar lo planteado por algunos académicos y sus producciones en torno a la permanencia académica y los factores causantes. Por ejemplo, Rizo (2010) sostiene que, entre otros factores, los académicos y pedagógicos generan dilación en los tiempos de formación. Tonconi (2010) sugiere una revisión detallada del sistema de créditos y los tiempos al interior de los estudiantes, como estrategia para evitar la saturación académica. Ragueyra (2014) aduce que los horarios y la administración académica, son causantes de limitaciones en el proceso educativo, especialmente en aquellos estudiantes con vínculos laborales.

Desde una mirada de la relación docente - estudiante, Shefi (2012), sustenta que los maestros influyen en las decisiones de los estudiantes, lo que puede llegar a generar incidencia en la continuación o cese de los estudios. Para Zarate y Socha (2010), las actitudes y comportamientos de los estudiantes 
al interior de las IES tienen gran incidencia en el proceso universitario. Pineda (2013) sostiene que el papel de los currículos es decisivo para garantizar un proceso académico exitoso, pues estos deben responder a los requerimientos de los estudiantes, la sociedad y el mundo académico.

Parra y Rodríguez (2014) sostienen que la generación de políticas de permanencia que se ajusten a las condiciones de la universidad pueden generar mejores indicadores. Este planteamiento es compartido por el Proyecto Alfa (2013), quienes consideran que el acompañamiento institucional genera éxito en los procesos académicos. Por otro lado, Torres (2012), considera que, entre otros factores, el bienestar universitario y la gestión institucional están ligados a la retención estudiantil. Finalmente, Natividad (2014) sostiene que los fundamentos teóricos y empíricos sobre la dilación académica, que puede también ser considerada como permanencia irregular, aún tienen muchas preguntas por resolver, a pesar de ser un fenómeno sumamente generalizado y con afectación directa sobre los estudiantes.

\section{Metodología}

La presente investigación es de tipo cuantitativo, toda vez que el método para la recolección de los datos se basa en la medición numérica de variables y en el análisis estadístico de las mismas. Lo anterior con el fin de poder establecer patrones de comportamiento en la población objeto del estudio (Hernández, Fernández y Batipsta, 2010). En este caso, el instrumento empleado para la recolección de la información fue la encuesta. Según Para, Trespalacios, Vázquez y Bello (2005), la encuesta es un instrumento propio de la investigación descriptiva que funciona a partir del diseño de preguntas cerradas elaboradas a priori y en las que se especifican las posibles respuestas del encuestado, quien finalmente hace parte de una muestra representativa de la población estudiada.

\section{Las variables del estudio}

El abordaje se hizo desde una perspectiva cuantitativa, buscando determinar el nivel de incidencia de seis factores de permanencia irregular, en la condición de irregulares de los estudiantes de pregrado presencial en la Facultad de Ciencias Contables, Económicas y Administrativas de la Universidad de la Amazonia en el periodo comprendido entre el 2010 - 2014. Las variables de estudio fueron los seis factores definidos por Gómez, Sánchez y Jiménez (2016) como los factores con mayor incidencia en el fenómeno de permanencia irregular: docentes, currículo, administración, rendimiento académico, medios educativos y bienestar universitario,

\section{Población y muestra}

La investigación tiene un corte no experimental, toda vez que buscó analizar los factores endógenos que causaron permanencia irregular en los estudiantes de los programas presenciales de la Facultad de Ciencias Contables, Económicas y Administrativas de la Universidad de la Amazonia en el periodo comprendido entre el 2010 al 2014. Es decir, tomó como caso de estudio a aquellos estudiantes que ingresaron en el primer periodo académico del 2010, y dado que los planes de estudio contemplaban 10 semestres, su graduación teóricamente tuvo que haber sucedido al finalizar el período 2014-II.

De este modo, los estudiantes vinculados a este ejercicio investigativo fueron aquellos que durante el periodo 2010-2014 cumplieron la condición de permanencia irregular. Es decir, aquellos que tuvieron vinculación permanente a la universidad durante el periodo del estudio, sin haber interrumpido el proceso y no se graduaron en los tiempos, en los programas de Contaduría Pública o Administración de Empresas, jornada diurna de la Facultad de Ciencias Contables y Administrativas.

En este orden, para definir el número de individuos de la población se tomó inicialmente a los 
estudiantes que ingresaron en el primer semestre académico de 2010 a cursar estudios universitarios en los programas académicos arriba mencionados. Esta generación conformó un universo total de 172 estudiantes de primer ingreso, excluyendo los que por transferencia externa ingresaron a cursar un semestre diferente al primero. Posteriormente, se procedió a excluir de este universo a los desertores, los cuales representaron el 55,2\% (95 estudiantes) quedando un total de 77 estudiantes en condición de permanencia irregular, que conformaron finalmente la población objeto del estudio.

La muestra para la realización del trabajo de campo fue del $46,75 \%$ de los estudiantes en condición irregular. Aunque se tenía como meta llegar al $100 \%$ de estos, fue imposible localizarlos, pues sus datos en las bases de datos institucionales estaban desactualizados y al momento de realizar su búsqueda (2015-II) no se encontraban matriculados oficialmente en la universidad.

\section{El método}

En consecuencia, la presente investigación se desarrolló a través de los siguientes pasos:

a Revisión documental: buscando un soporte teórico alrededor de las variables del estudio, se inspeccionaron tesis, artículos, informes de gestión, leyes, decretos y resultados de investigación, lo cual permitió ampliar la información conceptual acerca de ellas.

b Producción de los datos: la técnica utilizada para la recolección de la información base de análisis fue la encuesta personalizada. Esta se constituyó por un cuestionario de 16 preguntas, 10 cerradas y seis en la escala Likert. Las variables de estudio abordadas con este instrumento estuvieron relacionadas con la medición de la percepción de los estudiantes alrededor de la incidencia de los factores administración, currículo, medios educativos, bienestar universitario, docente y rendimiento académico sobre el fenómeno de la permanencia irregular. Luego, dentro de cada factor incidente, se identificaron y calificaron las posibles razones de su consideración o causas de la incidencia.

c Análisis de datos: la información fue tabulada en una base de datos en Excel. Luego se realizó el cruce de las variables y el establecimiento de relaciones entre ellas. Esto permitió identificar, a juicio de los estudiantes la incidencia de cada uno de los factores considerados en su permanencia irregular.

\section{Resultados y discusión}

Tal y como se detalló en el apartado de la metodología, la encuesta se aplicó a 36 estudiantes en condición de permanencia irregular, quienes, a su juicio, clasificaron los factores de la siguiente manera:

\section{Factor administración}

Fernández y Rosales (2014, p. 8) exponen que «la administración educativa es un conjunto de funciones que van orientadas hacia el ofrecimiento de servicios educativos efectivos y eficientes». Es decir, que esta se convierte en un puente de interacción entre la Universidad y, para este caso, los estudiantes. Por su parte, Martínez (2012), propone que:

La función de la administración en una institución educativa sería el planificar, diseñar, e implementar un sistema eficiente y eficaz para el logro de la enseñanza-aprendizaje en un entorno social en el que se imparte el servicio, para que responda a las necesidades de los alumnos y de la sociedad, es decir, responsabilizarse de los resultados de este sistema (p. 11). 
En tal sentido, tal y como lo afirmó Malpica (1980), la principal función de la administración educativa, y por ende de sus líderes, está relacionada con las políticas educativas desde su fase de incubación hasta la de evaluación de impacto, pues, tal y como lo indica Ramírez (2012), la administración educativa concibe a las instituciones de educación como una organización, la cual es susceptible a mejoras, cambios, transformaciones y reingenierías.

De esta manera, se indagó la posible incidencia de la administación educativa en la permanencia irregular de los estudiantes, encontrando que el 77.78\% (28 encuestados) de la población, no consideran a la administración como un factor generador de permanencia irregular. Sin embargo, el 22.22\% restante considera que si existe relación entre estas variables.

Entre los agentes causantes, a juicio de quienes respondieron de manera positiva (Figura 1), se encuentra la tardanza en los procesos de adición y cancelación de espacios académicos al inicio del semestre, dificultades para acceder a atención personalizada y poca información acerca de las opciones de grado ofertadas por la universidad. El anterior panorama, aunque pareciera tener poco impacto por las tendencias porcentuales, deja en evidencia la importancia de estrechar canales de comunicación efectivos, a fin de contrarrestar dinámicas unidireccionales, las cuales son generadoras de barreras (Acosta, 2008).

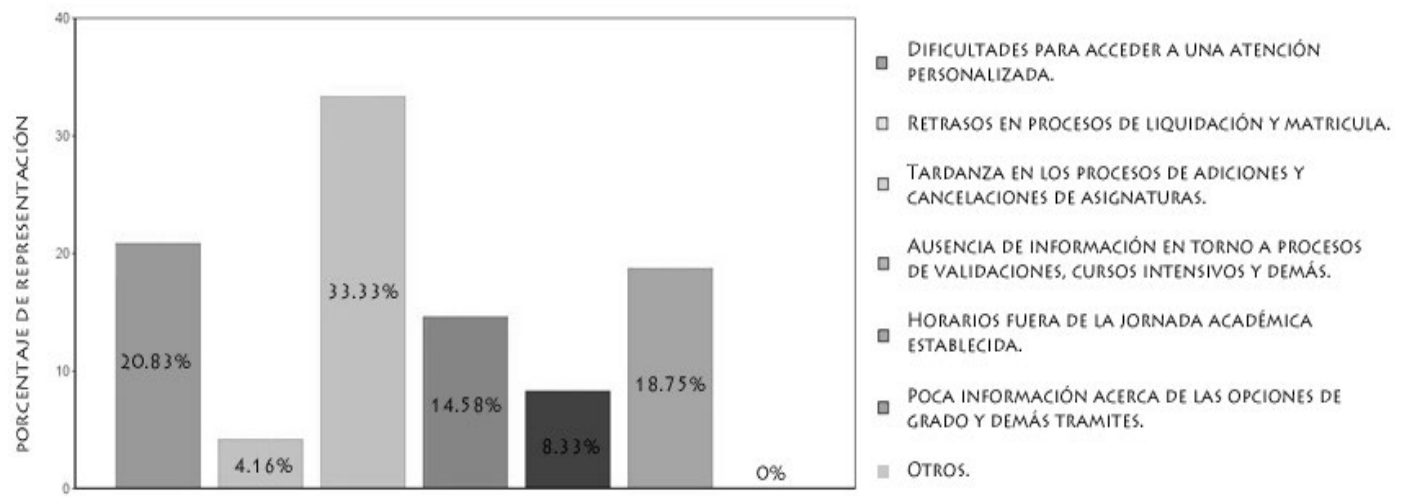

Figura 1. Agentes relacionados con la permanencia irregular en el factor Administración

\section{Factor currículo}

El Ministerio de Educación Nacional - MEN (2002), como máxima autoridad en los temas educativos del país, ha definido al currículo como «conjunto de criterios, planes de estudio, programas, metodologías, y procesos que contribuyen a la formación integral y a la construcción de la identidad cultural nacional, regional y local, incluyendo también los recursos humanos, académicos y físicos para poner en práctica las políticas y llevar a cabo el proyecto educativo institucional».

Para Guerra (2007), dada la gran diversidad de definiciones existentes en el mundo académico, es indispensable aceptar que el concepto de currículo tiene estrecha relación con las concepciones de educación, humanidad, cultura y desarrollo social. En línea con lo anterior, Terigi (1999) concibe al currículo como un factor clave para el éxito educativo, además afirma que:

a su valor para expresar y orientar el sentido formativa de la experiencia escolar se suma su capacidad para generar un proyecto de trabajo en cada escuela que haga posible que la distancia que siempre media entre la prescripción y las practicas se resuelva en términos de un enriquecimiento de las experiencias educativas de quienes asisten a las escuelas en calidad de alumnos (p. 13). 
Bajo este escenario, se indagó acerca de la incidencia del currículo en la permanencia irregular, encontrando que tan solo el $22.22 \%$ de los encuestados considera que este tiene relación con su permanencia irregular. Esta situación se evidencia en razones como inflexibilidad de los horarios, baja contextualización de algunos espacios académicos, excesos de prerrequisitos y poca actualización de los contenidos académicos, según expresaron los estudiantes que encontraron relación entre los fenómenos (Figura 2).

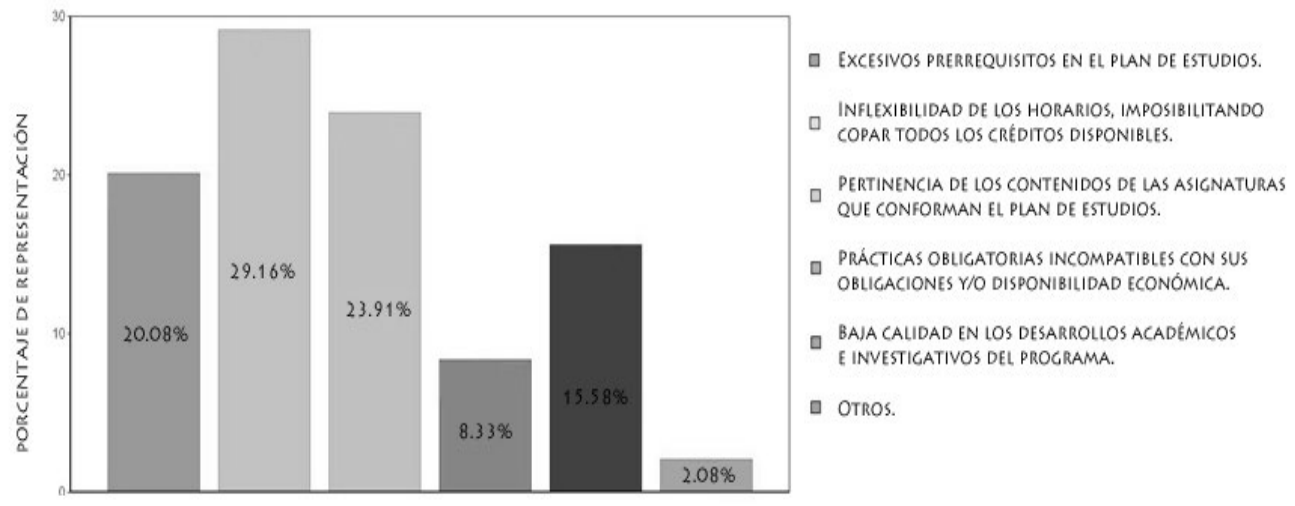

Figura 2. Agentes relacionados con la permanencia irregular en el factor Currículo

Los resultados de este acápite dejan sobre la mesa dos escenarios para el análisis. El primero relacionado con la inflexibilidad de los horarios, situación que se agudiza especialmente para los estudiantes de la jornada nocturna pues su presencialidad en la universidad se ve reducida. Sobre este particular es indispensable que los directivos y cuerpos colegiados asuman un riguroso proceso de planeación para la distribución de los espacios académicos, procurando que este no sea un agente que agudice el fenómeno de permanencia irregular.

En un segundo momento, se hace perentoria una revisión a los planes de estudio, pues estos como componentes importantes del currículo, sin desconocer la rigurosidad académica e investigativa que los debe caracterizar, máxime en el nivel de educación superior, deben ser un derrotero que cautive a los estudiantes, estructurado de tal manera que les permita visibilizar su evolución disciplinar, acercándolo cada vez más a la profesionalización. Bajo este escenario, conviene retomar lo planteado por Nolla (2001), quien sostiene que el plan de estudios:

(...) juega un papel fundamental en las instituciones educativas, pues constituye la guía de acción a profesores y estudiantes en la realización y control del proceso enseñanza-aprendizaje a partir de los objetivos profesionales o de nivel que se desean lograr y ofrece las orientaciones de cómo debe desarrollarse dicho proceso, dejando siempre la posibilidad de que el profesor programe los contenidos de acuerdo con su creatividad y a las motivaciones e intereses de los estudiantes, que permita la inclusión de las novedades de la ciencia y la adecuación al contexto y necesidades de la institución en que se desarrolla (p. 150).

\section{$3 \quad$ Factor rendimiento académico}

Los conceptos, definiciones y perspectivas sobre el rendimiento académico son, además de abundantes, diversos según la posición y percepción de los diferentes autores. Por ejemplo, desde una perspectiva integral, Montes y Lerner (2010) lo definen como: 
La relación entre el proceso de aprendizaje, que involucra factores extrínsecos e intrínsecos al individuo, y el producto que se deriva de él, expresado tanto en valores predeterminados por un contexto sociocultural como en las decisiones y acciones del sujeto en relación con el conocimiento que se espera obtenga de dicho proceso (p. 15).

Por su parte, Navarro (2003, p. 12) lo define como «un constructo susceptible de adoptar valores cuantitativos y cualitativos, a través de los cuales existe una aproximación a la evidencia y dimensión del perfil de habilidades, conocimientos, actitudes y valores desarrollados por el alumno en el proceso de enseñanza aprendizaje». Esa posibilidad de representación en formas cualitativas o cuantitativas ha conllevado a que, ya sea por practicidad, efectividad, ausencia de rigurosidad o simplemente por tecnicismo, el rendimiento académico se exprese de forma cuantitativa, privilegiando las escalas numéricas como forma de expresión, dejando atrás aspectos cualitativos que desbordan la rigidez de una cifra (Erazo, 2012).

Al indagar en los estudiantes en condición de permanencia irregular, se hizo evidente que estos relacionan su rendimiento académico con las calificaciones obtenidas en los diferentes espacios académicos. La encuesta evidenció que el $47.22 \%$ considera que sí existe relación entre su rendimiento académico y su condición de permanencia irregular. Esto podría verse, al menos desde quienes respondieron positivamente, como un ejercicio de autocrítica, pues los estudiantes reconocen ausencia de interés, poca dedicación a las actividades extra-clase y la baja asistencia a las sesiones académicas como los principales generadores de su bajo rendimiento académico (Figura 3).
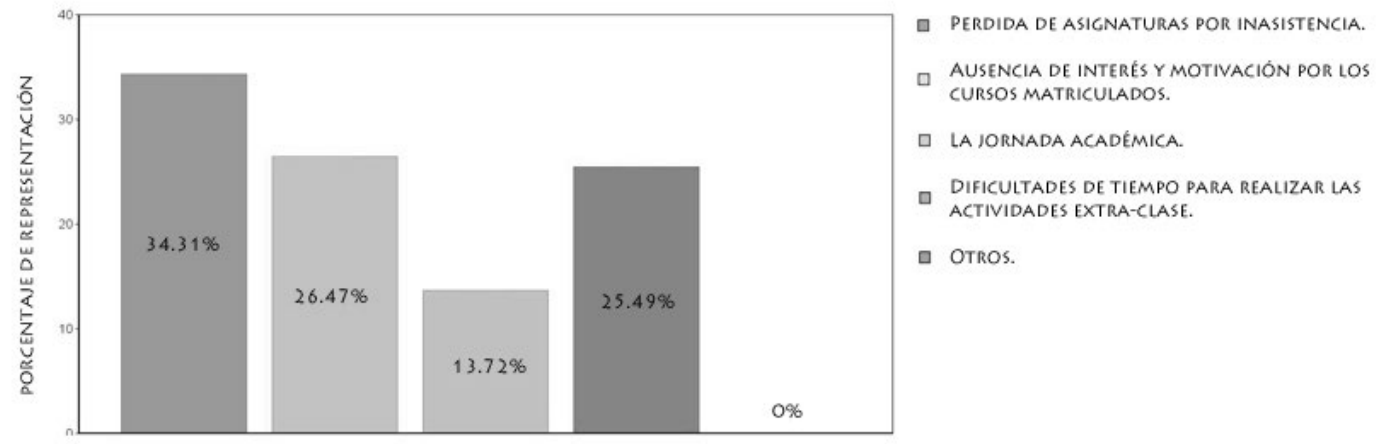

Figura 3. Agentes relacionados con la permanencia irregular en el factor Rendimiento Académico

Finalmente, por ser un tema de interés colectivo, se hace necesaria la intervención de todos los actores de la comunidad académica, a fin de mejorar el rendimiento académico de los estudiantes, no solo desde una posición cuantitativa (mejores calificaciones), sino atendiendo variables cualitativas como motivación, compromiso y disciplina, las cuales son garantía de un exitoso proceso de formación académico. Sobre el particular, Fenollar, Cuestas, y Román, (2008), en su apartado de conclusiones exponen que:

Aconsejamos a los profesores que realicen un seguimiento cercano de la evolución en las habilidades y conocimientos de sus alumnos, corrigiendo, en su caso, las posibles desviaciones. Es decir, que se les transmita la idea de que llevan una dirección adecuada hacia el aprendizaje y la comprensión de los conceptos de la asignatura (cuando así sea), pero también que es más provechosa y conveniente a largo plazo esta orientación al aprendizaje, ya que consolida conocimientos para su futuro (p. 18). 


\section{$4 \quad$ Factor medios educativos}

Un medio educativo es cualquier componente que estimule el aprendizaje» (Bravo, 1998, p. 3). Por su parte, Marqués $(2000$, p. 1) afirma que «recurso educativo es cualquier material que, en un contexto educativo determinado, sea utilizado con una finalidad didáctica o para facilitar el desarrollo de las actividades formativas. Los recursos educativos que se pueden utilizar en una situación de enseñanza y aprendizaje pueden ser o no medios didácticos.

En tal sentido, los medios educativos, siguiendo a Blázquez y Lucero (2002, p. 186), son

cualquier recurso que el profesor prevea emplear en el diseño o desarrollo del currículo (por su parte o la de los alumnos) para aproximar o facilitar los contenidos, mediar en las experiencias de aprendizaje, provocar encuentros o situaciones, desarrollar habilidades cognitivas, apoyar sus estrategias metodológicas, o facilitar o enriquecer la evaluación.

Al indagar sobre la relación entre los agentes «medios educativos» y «permanencia irregular», se encontró que tan solo 5 de los encuestados, que representan el 13.89\% de la población, consideran que existe relación entre estos dos agentes. Estos manifestaron que la baja utilización de las plataformas virtuales, poco acceso a softwares especializados y las restricciones para acceder a las salas de conferencias, se constituyeron en las principales razones para tipificar la mencionada relación (Figura 4).

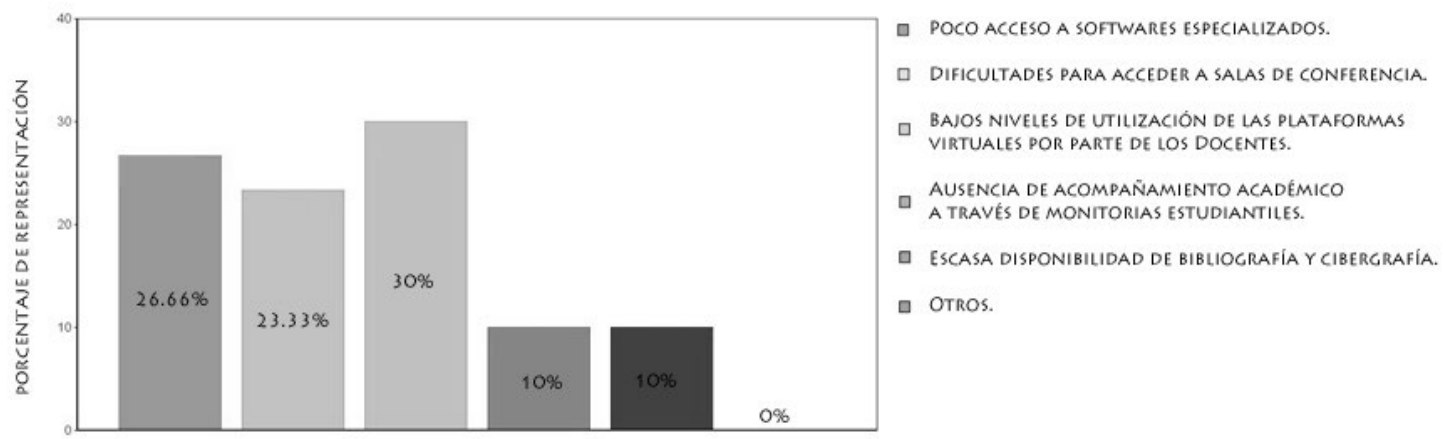

Figura 4. Agentes relacionados con la permanencia irregular en el factor Medios Educativos

El anterior panorama genera un parte de tranquilidad para la institución y los maestros, pues, tal y como lo indica el MEN (2005), la inclusión de medios educativos que dinamicen el proceso de enseñanza-aprendizaje es una prioridad en los sistemas educativos, dado que estos aseguran, de cierta manera, el éxito en la interacción de la triada estudiantes-conocimiento-docentes (Sánchez et al. 2016).

\section{$5 \quad$ Factor bienestar universitario}

Los desarrollos académicos e investigativos propios de la educación superior deben desarrollarse en condiciones óptimas para los integrantes de la comunidad académica, especialmente para los estudiantes quienes, por los procesos propios de sus responsabilidades, se ven sometidos a extensas 
jornadas de trabajo. Adicionalmente, la universidad, especialmente para los estudiantes de la jornada diurna, se convierte en un espacio de habitación permanente, lo que demanda entonces la adecuación de condiciones de calidad.

En tal sentido, López y Díaz (2009, p. 80), respecto al bienestar universitario expresan que «su quehacer desde la formación integral, en busca de un mejoramiento de la calidad de vida de la comunidad universitaria», ratificándolo entonces su responsabilidad con la cómoda estancia, para este caso, de los estudiantes al interior de la institución. Al indagar sobre la relación de este factor con el fenómeno de permanencia irregular, se encontró que para la mayoría de los encuestados (97.22\%) no existe una relación entre los dos factores. Estos resultados evidencian una solidez en la política de bienestar universitario de la institución, situación que es atribuible, según el cuerpo directivo de la institución, a los crecientes indicadores de cobertura en subsidio alimentario, incremento en el apoyo a los equipos deportivos, construcción de escenarios deportivos en la institución y la solidez del talento humano encargado de este fin. Este abanico de posibilidades se generó gracias a recursos del CREE y la estampilla pro Universidad de la Amazonia. De esta manera, el factor Bienestar Universitario, a juicio de los encuestados, presenta una baja relación con el fenómeno de permanencia irregular. Empero, para quien respondió de manera positiva, las causas están relacionadas con los factores descritos en la Figura 5:

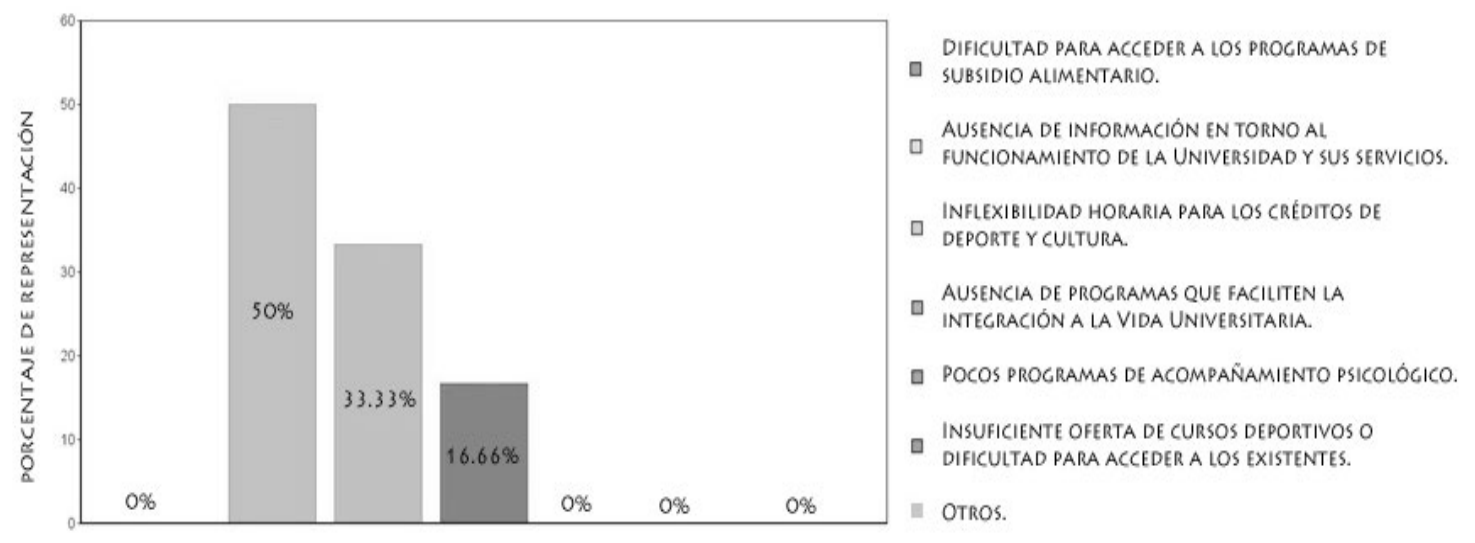

Figura 5. Agentes relacionados con la permanencia irregular en el factor Bienestar Universitario

\section{Factor Docente}

Las nuevas tendencias pedagógicas plantean al docente como un actor estratégico del proceso educativo, quien, lejos del rol dominante e impositivo de los modelos tradicionales, se convierte en un facilitador de las dinámicas del proceso de enseñanza-aprendizaje. Sobre el particular, Mas (2011) expresa que:

En lo referente a la función docente, la responsabilidad del profesor universitario traspasa los límites del aula donde desarrolla el acto didáctico (fase interactiva); también debemos considerar el diseño y planificación de dicha formación (fase preactiva), la evaluación de las competencias adquiridas y/o desarrolladas y, como no, la contribución de dicho profesional a la mejora de la acción formativa desarrollada y su participación en la dinámica académico-organizativa de su institución. (p. 199). 
Aunado a lo anterior, Torres (2012, p. 84) sostiene que

el rol del docente está delimitado por su función orientadora dentro de un establecimiento educativo, para el caso,- una Institución de Educación Superior-, de un proceso de formación, enseñanza y aprendizaje de los estudiantes, acorde con las expectativas sociales, culturales, éticas y morales de la familia y la sociedad

ratificando el rol protagónico de los docentes en el éxito del proceso educativo. De esta manera, se indagó acerca de la relación existente entre el factor docente y la permanencia irregular, encontrando que $57.78 \%$ de los encuestados considera que existe relación entre estas dos variables. Las causas de esta relación, para quienes respondieron positivamente, están ligadas a la poca actualización de planes de trabajo y de desarrollo de curso, las estrategias didácticas y pedagógicas utilizadas en el proceso de enseñanza-aprendizaje, deficiencias en la relación humana docente-estudiante y la rigidez de los horarios para las asesorías extra clase (Figura 6).

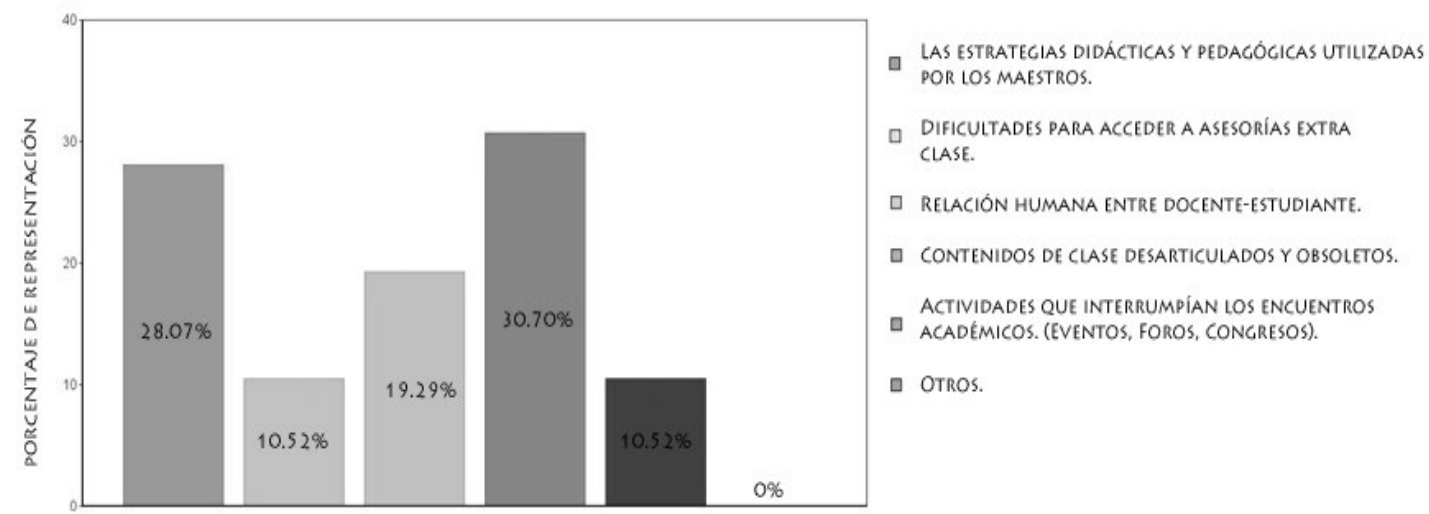

Figura 6. Agentes relacionados con la permanencia irregular en el factor Docencia

Estos resultados avizoran al menos tres aristas de análisis. El primero relacionado con la importancia de actualización y reingeniería de los espacios académicos, pues si bien existen conceptos que pueden ser estándar, la contextualización y aplicabilidad del saber debe ser una estrategia liderada por los docentes. En un segundo momento, tal y como afirma Miller (2002), la instituciones debe generar planes permanentes de formación docente, a fin de permitir espacios para el perfeccionamiento de su ejercicio profesional.

Finalmente, el plano de las relaciones humanas, siguiendo a Covarrubias y Piña (2004), tiene alta influencia en los procesos pedagógicos, autor que concluye que «la relación maestro-alumno como uno de los factores del contexto educativo que más repercute en la calidad del aprendizaje» (p. 80). Esta situación se convierte en un insumo para el desarrollo de investigaciones y procesos de autoevaluación que permitan tener mayores claridades sobre esta variable.

\section{Conclusiones}

La lucha contra la permanencia irregular es un naciente reto para las universidades, pues este fenómeno de tímidos análisis e investigaciones a la fecha, afecta significativamente a los sistemas de educación. Además, cuestiona las acciones de seguimiento y control al interior de los claustros 
educativos, situación que para los entes rectores en el tema, son una clara muestra de inefectividad de las instituciones y un atentado contra la calidad. De esta manera, se hace necesario que la comunidad académica, especialmente quienes se dedican a los procesos de investigación, inicien una serie de intervenciones sobre el tema, generando así un mayor marco teórico que permita una mejor tipificación del fenómeno, siendo este el primer paso para su erradicación.

A la luz de los resultados encontrados, se evidenció que los factores rendimiento académico y docente son los que, según los estudiantes, representan mayor incidencia en su permanencia irregular. Esta situación invita a una profunda reflexión por parte de los directivos, cuerpos colegiados y planta docente, dado que, de manera paradójica, son los agentes con mayor relación en el dúo docenteestudiante. Es por lo anterior, que las estrategias de intervención son prioritarias, pues la proliferación de este fenómeno amenaza la calidad de los programas, sin mencionar todos los elementos adicionales que se generan por ser una institución pública.

En un segundo momento, los factores Administración y Currículo, pese a tener una participación porcentual poco representativa, no pueden ser descartados, en razón a que estos tienen una estrecha relación en el quehacer docente y por ende en los desarrollos académicos, pues son génisis de las transformaciones requeridas por la actual sociedad del conocimiento, la cual obliga a las instituciones a someterse a constantes procesos de reingeniería. De esta manera se recomienda a las instituciones prestadoras de servicios educativos la evaluación constante de estos agentes, además, la vinculación de agentes actores estratégicos como el sector productivo, gobierno, organizaciones civiles, redes académicas e investigativas en los procesos de transformación de sus mallas curriculares, acercando sus contenidos a las realidades sociales.

Finalmente, los factores Medios Educativos y Bienestar Universitario son los menos representativos dentro del estudio, situación que puede estar atribuida a que la jornada académica que impera en la Facultad es la nocturna. Dadas las condiciones propias de su desarrollo, la permanencia de los estudiantes es limitada, circunscribiéndose a un máximo de 4 horas presenciales. Adicionalmente, la popularización de las herramientas tecnológicas básicas pueden disminuir la demanda de espacios dotados por parte de la universidad. Sin embargo, bajo una dinámica de mejoramiento constante y calidad en el servicio educativo, la institución debe generar esfuerzos por mejorar las condiciones, garantizando niveles de bienestar en la permanencia de los estudiantes.

\section{Bibliografia}

Acosta C, A. (2008). La comunicación como vía hacia una educación consensual. Tesis de pregrado en Comunicación Social. Pontificia Universidad Javeriana. Bogotá, Colombia.

Ambroggio, G. (2012). El primer año en la universidad y la permanencia en la carrera. Cuadernos de Educación, 1(1), 133-143

Blázquez, F., y Lucero, M. (2002). Los medios y recursos en el proceso didáctico. En Medina, A. \& Salvador, F. Didáctica General (pp. 185- 218). Madrid: Pearson Educación.

Bravo R, JL. (1998). Los medios didácticos en la enseñanza universitaria. Madrid, España.

Carrión, D., Sanmartín, S., Fernández, MS. (2014). Facilitadores y obstáculos en las trayectorias de los Estudiantes en la Universidad Nacional General de Sarmiento. Disponible en: http://iice. institutos.filo.uba.ar/sites/iice.institutos.filo.uba.ar/files/10-Niveles\%20educativos_00000000. pdf

Congreso de la Republica de Colombia. (1992). Ley 30 de 1992 «Por la cual se organiza el servicio público de la educación superior». Santafé de Bogotá, Diario Oficial 40700 de Diciembre 29 de 1992. 
Constitución Política de Colombia. (1991). Artículo 67.

Covarrubias P, P., y Piña R. (2004). La interacción maestro-alumno y su relación con el aprendizaje. Revista Latinoamericana de Estudios Educativos, vol. XXXIV, núm. 1. Centro de Estudios Educativos, A.C. Distrito Federal, México.

Cuchacovich G, IA. (2011). Retención y movilidad universitaria: Evaluación de Impacto de una Política. Tesis de Maestría. Pontificia Universidad Católica de Chile, Santiago de Chile (Chile). Departamento Nacional de Planeación (DNP). (2015). Plan Nacional de Desarrollo 2014-2018. Imprenta Nacional de Colombia. Bogotá, Colombia.

Dupéré, V., Leventhal, T., Dion, E., Crosnoe, R., Archambault, I., \& Janosz, M. (2014). Stressors and turning points in high school and dropout: A stress process, life course framework. Review of Educational Research. Advance online publication.

Erazo, OA. (2012). El rendimiento académico, un fenómeno de múltiples relaciones y complejidades. Revista Vanguardia Psicológica, volumen 2, numero 2. Universidad Manuela Beltrán. Bogotá, Colombia.

Fenollar Q, P., Cuestas F, PJ., y Román N, S. (2008). Antecedentes del rendimiento académico: aplicación a la docencia en marketing. Revista Española de Investigación de Marketing ESIC, volumen 12 , numero 2 ..

Fernández, S., y Rosales, M. (2014). Administración educativa: la planificación estratégica y las prácticas gerenciales integrando la tecnología, su impacto en la educación. Congreso Iberoamericano de Ciencia, Tecnología, Innovación y Educación. Buenos Aires, Argentina.

Gómez, C., Sánchez C., y Jiménez, Z. (2016). Factores endógenos relacionados con la permanencia irregular en las IES, una aproximación conceptual. Revista En-Contexto 5, 285-306.

Gómez, C., Sánchez C., y Rivera C. (2016). Programa jóvenes en acción en la Universidad de la Amazonia: una lectura desde los actores en cuestión. Revista Cooperativismo y Desarrollo 24 (108), 157-173.

Guerra M, JW. (2007). Proceso de Diseño Curricular. Proyecto Formación de Directivos Docentes en Antioquia. SEDUCA - FUNLAM. Medellín, Colombia.

Hernández, R., Fernández, C., Batipsta, M.P. (2003). Metodología de la Investigación. Quinta Edición. México: MGraw Hill.

López D, EL., y Díaz D, SJ. (2008). Representaciones sociales sobre bienestar universitario de los representantes estudiantiles de la Universidad del Quindio. Tesis de Maestría en Educación y Desarrollo Humano. Universidad de Manizales. Caldas, Colombia.

Malpica, C. (1980). La administración de la educación y sus relaciones con la planificación y con la investigación. Instituto Internacional de Planeamiento de la Educación. Paris, Francia.

Marqués, P. (2001). Los medios didácticos y los recursos educativos. Disponible en: https://graphos.wikispaces.com/file/view/ LOS+MEDIOS+DID\%C3\%81CTICOS+Y+LOS+RECURSOS+EDUCATIVOS.pdf, consultado el 20.12.2016

Martínez A, L. (2012). Administración educativa, primera edición. RED TERCER MILENIO S.C. Impreso en México.

Mas T, O. (2011). El profesor universitario: sus competencias y formación. Revista Profesorado, volumen 15, numero 3. Universidad de Granada. Granada, España.

Melo, B., Ramos, F., Hernández, S. (2014). La Educación Superior en Colombia: situación actual y análisis de eficiencia. Borradores de Economía, Número 108. Banco de la Republica de Colombia. Bogotá, Colombia. 
Miller, E. (2002). Políticas de formación docente en la mancomunidad del Caribe. Formación docente: un aporte a la discusión. La experiencia de algunos países. Oficina Regional de Educación de la UNESCO. Impreso en Chile.

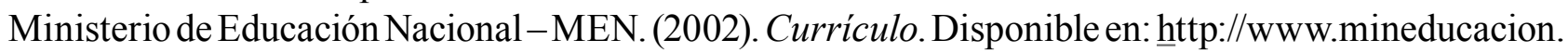
gov.co/1621/article-79413.html, consultado el 19.12.2016

Ministerio de Educación Nacional - MEN. (2005). Ser maestro hoy. Disponible en: http://www. mineducacion.gov.co/1621/propertyvalues-31232_tablero_pdf.pdf, consultado el 10.12.2016

Ministerio de Educación Nacional - MEN. (2009). Deserción estudiantil en la educación superior colombiana. Imprenta Nacional de Colombia. Viceministerio de Educación Superior. Bogotá, Colombia.

Ministerio de Educación Nacional - MEN. (2013.). Política y estrategias para incentivar la permanencia y graduación en educación superior 2013-2014. Viceministerio de Educación Superior. Bogotá, Colombia.

Misas, G. (2004). La educación superior en Colombia: análisis y estrategias para su desarrollo. Universidad Nacional de Colombia. Bogotá, Colombia.

Montes G, IC., Lerner M, J. (2010). Rendimiento académico de los estudiantes de pregrado de la Universidad EAFIT. Dirección de Planeación, Universidad EAFIT. Bogotá, Colombia.

Natividad S, LA. (2014). Análisis de la Procrastinación en Estudiantes Universitarios. Tesis doctoral. Universidad de Valencia, Valencia (España).

Navarro, R. (2003). El rendimiento académico: concepto, investigación y desarrollo. REICE - Revista Electrónica Iberoamericana sobre Calidad, Eficacia y Cambio en Educación, volumen 1, numero 2. Disponible en: http://www.ice.deusto.es/RINACE/reice/volln2/Edel.pdf, consultado el 20.12.2016

Nolla C, N. (2001). Los planes de estudio y programas de las especialidades médicas. Revista Cubana de Educación Medica Superior, volumen 15, número 2. Ciudad de la Habana, Cuba.

Organización de las Naciones Unidas para la Educación, la Ciencia y la Cultura - UNESCO. (2014). El desarrollo sostenible comienza por la educación. Impreso en Francia.

Parra, DC., Rodríguez, LA. (2014). Factores que inciden en la Permanencia Académica de los Estudiantes de la Universidad Nacional Abierta y a Distancia "UNAD” - CEAD Facatativá. Tesis de pregrado. Facatativá, Cundinamarca (Colombia).

Pineda B, C. (2013). Compromiso estudiantil y desempeño académico universitario: Comprobando el vínculo. Universidad de la Sábana, Chía (Colombia).

Proyecto ALFA. (2013). Hacia La Construcción Colectiva De Un Marco Conceptual Para Analizar, Predecir, Evaluar Y Atender El Abandono Estudiantil En La Educación Superior. Medellín (Colombia).

Ragueyra E, MG. (2014). Aprendizajes sobre la población estudiantil que no continúa y el derecho a la educación superior. Disponible en: http://www.alfaguia.org/www-alfa/images/ponencias/ clabesIII/LT_1/ponencia_completa_8.pdf.

Ramírez C, CA. (2012). La Gestión Educativa (GE) en la educación básica y media oficial de Manizales: un análisis desde las teorías administrativas y organizacionales. Tesis de Maestría en Administración. Universidad Nacional de Colombia, sede Manizales. Caldas, Colombia.

Rizo-Bonilla, Y.A. (2010). Factores que afectan las distintas formas de culminación de estudios que realizan los(as) estudiantes de la carrera administración de empresas, de la modalidad por encuentro dominical de la universidad de las américas (ulam) Managua. Revista Catedra, 1(1)1-127. Recuperado en http://revistacatedra.unan.edu.ni/index.php/investigaciones/article/ 
view/334/304

Sánchez, V., Gómez, C y Polanía, L. (2016). La Educación Superior en Colombia: una cuestión de calidad, no de cantidad. Revista Criterios, 23(1), 141-168.

Sánchez C, V., Gómez C, CA., Ortiz P, D., Clavijo G, TA., y Váquiro R, LP. (2016). Percepción Social Importancia del Inglés e Inclusión de Videojuegos como Herramienta de Aprendizaje. Revista Amazonia Investiga, 5(8), 58-66

Shefi, Y. (2012). La contribución de las relaciones docente-alumno de perseverancia, para la prevención de deserción escolar y motivación para el cambio en las actitudes de los estudiantes en "segunda oportunidad" en Escuela Superior. Napoc, Romania: Universidad Cluj.

Terigi, F. (1999). Curriculum. Itinerarios para aprehender un territorio. Editorial Santillana. Buenos Aires, Argentina.

Trespalacios, J; Vásquez, R., Bello, L. (2005). Investigación de mercados: métodos de recogida y análisis de la información para la toma de decisiones de marketing. España: Ediciones Paraninfo.

Tonconi Q, J. (2010). Factores que influyen en el Rendimiento Académico y la Deserción de los Estudiantes de la Facultad de Ingeniería Económica de la UNA-PUNO, periodo 2009. En: Revista Cuadernos de Educación y Desarrollo, volumen 2, número 11. Universidad de Málaga (España).

Torres G, LE. (2012). Retención estudiantil en la educación superior: revisión de la literatura y elementos de un modelo para el contexto colombiano. Editorial Pontificia Universidad Javeriana, primera edición. Bogotá (Colombia).

Torres R, AM. (2012). Rol y características del liderazgo del docente en la Educación Superior a distancia en Colombia. Tesis de doctorado en Educación. Universidad de Zaragoza. Zaragoza, España.

Universidad Abierta para Adultos - UAPA. (2009). Deserción en las instituciones de educación superior a distancia en América Latina y el Caribe. Ediciones UAPA. Impreso en República Dominicana.

Zarate, R., Socha, CM. (2010). Estudio sobre las motivaciones de deserción estudiantil en la Universidad Industrial de Santander. Disponible en http://www.alfaguia.org/alfaguia/files/1320183574_24. pdf. 